\title{
Characterization of Wafer-Level Thermocompression Bonds
}

\author{
Christine H. Tsau, S. Mark Spearing, Member, ASME, and Martin A. Schmidt, Fellow, IEEE
}

\begin{abstract}
Thermocompression bonding joins substrates via a bonding layer. In this paper, silicon substrates were bonded using gold thin films. Experimental data on the effects of bonding pressure (30 to $120 \mathrm{MPa})$, temperature $\left(260\right.$ and $\left.300{ }^{\circ} \mathrm{C}\right)$, and time ( to $90 \mathrm{~min}$ ) on the bond toughness, measured using the four-point bend technique, are presented. In general, higher temperature and pressure lead to higher toughness bonds. Considerable variation in toughness was observed across specimens. Possible causes of the nonuniform bond quality were explored using finite element analysis. Simulation results showed that the mask layout contributed to the pressure nonuniformity applied across the wafer. Finally, some process guidelines for successful wafer-level bonding using gold thin films are presented.

[1170]
\end{abstract}

Index Terms-Bond characterization, thermocompression bonding, wafer bonding.

\section{INTRODUCTION}

W AFER bonding has enhanced fabrication capabilities for microelectromechanical systems (MEMS). While $\mathrm{Si}$ fusion and anodic bonding may be the most prominent wafer bonding methods, they are not the only options for joining substrates. Wafers can be effectively bonded together by using bonding layers such as polymers, solders and thin film metals. The incorporation of metals, especially those that are conductive, in conjunction with through-wafer via technology, also presents opportunities for packaging applications. Hybrid structures consisting of devices fabricated separately on different substrates can also be created by wafer-level bonding.

Metallic bonds form when the distances between the two substrates are so small that it becomes energetically favorable for surfaces to coalesce in order to eliminate the interfacial energy. The surfaces can be brought together by the application of pressure. Due to surface roughness, bonds initially form where the surfaces touch at asperity contacts. As the materials deform under pressure, the asperity heights decrease, and more areas are brought into contact. At room temperature, relatively high pressures are needed for interatomic attraction to overcome surface

Manuscript received October 14, 2003; revised April 12, 2004. This work was supported by DARPA (F30602-97-C-0127), SRC (2001-MJ-881), the Singapore-MIT Alliance (SMA MOU 11/6/98), and an SRC Graduate Fellowship. Subject Editor D. Cho.

C. H. Tsau is with the Media Lab, Massachusetts Institute of Technology, Cambridge, MA 02139 USA (e-mail: chtsau@mit.edu).

S. M. Spearing was with the Department of Aeronautics and Astronautics, Massachusetts Institute of Technology, Cambridge, MA 02139 USA. He is now with the School of Engineering Sciences, University of Southampton, Southampton SO17 1BJ, U.K.

M. A. Schmidt is with Microsystems Technology Laboratories, Department of Electrical Engineering and Computer Science, Massachusetts Institute of Technology, Cambridge, MA 02139 USA.

Digital Object Identifier 10.1109/JMEMS.2004.838393 asperities in metals [1]. But dislocation mobility and diffusion increases with temperature, resulting in softening of the metal and viscoplastic deformation. Therefore, the pressure requirement can be offset by increasing the processing temperature. The technique of simultaneous application of pressure and temperature during the bonding process is known as thermocompression bonding. This process is essentially identical to diffusion bonding, which is used extensively for joining metallic components at the macro scale.

Almost any metal can be bonded via thermocompression. However, the requisite pressure and temperature may not be in a practical range for all metals. For oxidizable metals, such as solder or even $\mathrm{Cu}$, the bonding surfaces must be treated to remove the oxide that would impede bond formation. As a noble metal, gold is an ideal bonding material, and a good example of thermocompression bonding is gold wire bonding. However, it is not a batch process. Most studies of gold thermocompression bonding involve chip-level bonds [2]-[4]. Few have studied large area, wafer-level bonds. Furman and Mita described bonding of 60000 pads on $127 \times 127 \mathrm{~mm}^{2}$ ceramic substrates [5]. The pressure, which ranged from 0.689 to $2.76 \mathrm{MPa}$, was applied for $3 \mathrm{hr}$, with $1 \mathrm{hr}$ at the peak temperature of either 400 or $375^{\circ} \mathrm{C}$. These are the pressures that were applied to the substrate. Since the actual bonding area was some fraction of the bond pads, each $150 \mu \mathrm{m} \times 450 \mu \mathrm{m}$, the actual bonding pressure on the gold was greater than the peak pressure reported. At the wafer-level, Drost $e t$ al. found a pressure of $0.06 \mathrm{MPa}$ was insufficient for continuous bond formation: bonds were highly localized and on the micrometer scale [6]. At the chip-level, 4 $\mu \mathrm{m}$ gold films were bonded for $1 \mathrm{~min}$ with varying temperatures and pressures on the gold, from 350 to $450{ }^{\circ} \mathrm{C}$ and 0.32 to $17.86 \mathrm{MPa}$, respectively. An increase in yield was observed for pressures up to $1 \mathrm{MPa}$; further increases in pressure or temperature showed little benefit. However, additional bonding time was reported to improve bond homogeneity [6].

The present paper attempts to perform a more systematic characterization of the thermocompression bonding process at the wafer-level, focusing on temperatures less than or equal to $300{ }^{\circ} \mathrm{C}$. Among the host of variables, the parameters that brings surfaces into close proximity for bonding to occur are believed to have the most impact on the bond quality. The effect of bonding layer thickness was explored previously [7]. This paper discusses the effects of bond pressure, temperature, and time on the measured bond toughness. Then, results of finite element simulation on the bond pressure distribution are presented. Finally, guidelines on the processing of thermocompression bonds are provided. 


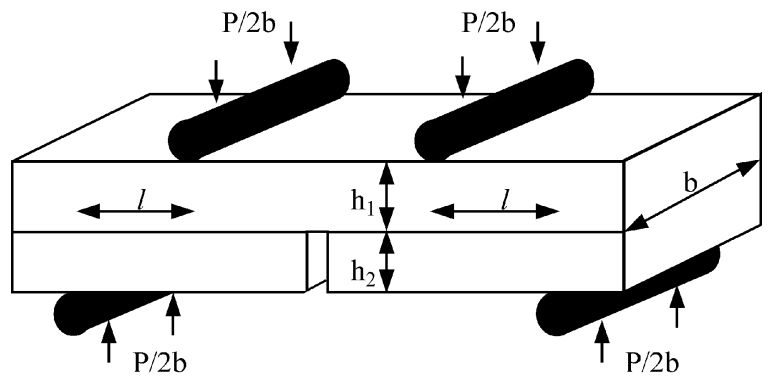

Fig. 1. Schematic of a typical four-point bend specimen.

\section{FRACTURE TOUGHNESS}

The fracture toughness of a bonded interface provides a quantitative measurement of the bond quality. This is based on the idea that crack propagation changes the potential energy of the material. Fracture creates free surfaces, with an associated surface energy $\gamma$. That energy is balanced by the external load that drove the crack propagation in the first place, and a reduction in the stored elastic strain energy in the material. The change in the total potential energy with area is known as the strain energy release rate; the crack propagates only when the critical strain energy release rate of the material $\mathcal{G}_{c}$ is reached.

In the wafer bonding literature, the bond integrity is often measured using the crack-opening method [8]. The test creates a crack in the bonded wafer by insertion of a razor blade at the edge of the interface. The crack length, which is measurable in the infrared (IR), is then used to calculate the surface energy. Its simple implementation makes it a very attractive test technique. However, the crack opening is difficult to induce when the bond toughness is high, which often makes quantitative measurements impossible. Moreover, the technique is inapplicable when the bonding interface is opaque in the IR, such as gold.

In this paper, the bond quality was measured using a fourpoint bend-delamination technique [9]. The specimen is a beamlike structure with a width-wise notch in the bottom beam, as shown in Fig. 1. A constant moment condition is established between the inner rollers. Once the crack length exceeds the beam thickness, the strain energy release rate $\mathcal{G}$ is independent of the crack length, as given by the following equation [9]

$$
\mathcal{G}=\frac{1.5\left(1-\nu^{2}\right)}{E}\left(\frac{P l}{b}\right)^{2}\left(\frac{1}{h_{1}^{3}}-\frac{1}{\left(h_{1}+h_{2}\right)^{3}}\right)
$$

where $P$ is the applied load, $l$ is the distance between the inner and outer rollers, $b$ is the width of the specimen, and $E, \nu, h_{1}$ and $h_{2}$ are the Young's modulus, the Poisson's ratio, and the thickness of the unnotched wafer and notched wafer, respectively. Equation (1) shows $\mathcal{G}$ as functions of the specimen geometry and applied load, both of which can be measured quite accurately by commercially available equipment. If $P_{c}$, the critical load at which crack propagation occurs, is used in (1), then $\mathcal{G}$ becomes $\mathcal{G}_{c}$, the critical strain energy release rate.

\section{EXPERIMENTAL PROCEDURE}

The details of the fabrication process have been reported in [7], so only a brief outline is given here. Each wafer pair consisted of two $4^{\prime \prime} \mathrm{Si}$ wafers, one of which is a double side (a)
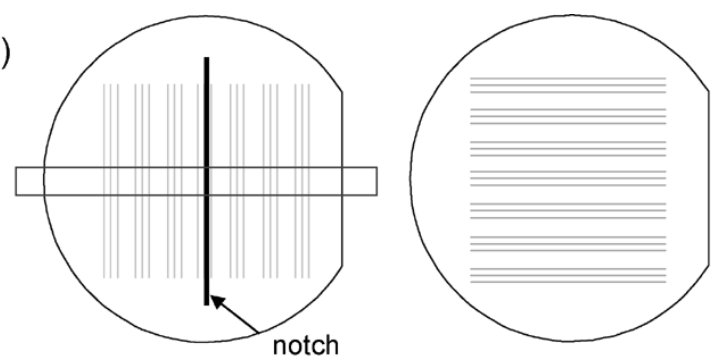

(b)

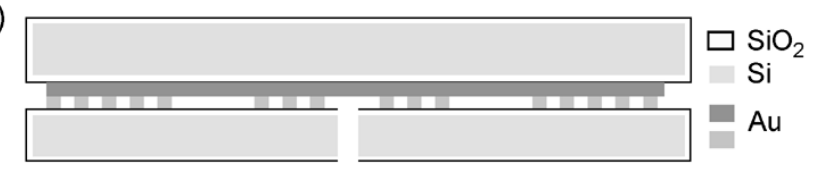

(c)

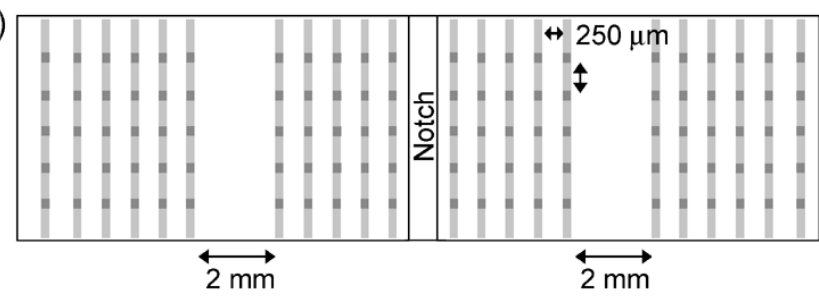

Fig. 2. Illustration of the specimen geometry. (a) Wafer pair where the metal lines will be bonded in orthogonal directions to one another. (b) Cross section of the specimen outlined in (a) showing the portion within in the inner rollers. (c) Top view of the bottom wafer, with the pad formation highlighted.

polished (DSP) wafer. Following the formation of a $500 \mu \mathrm{m}$ wide, $60-70 \mu \mathrm{m}$ deep trench in the DSP wafer via $\mathrm{KOH}$ and anisotropic etching, approximately $300 \mathrm{~nm}$ of thermal oxide was grown on both substrates. Metals were then e-beam deposited on resist patterned wafers: $10 \mathrm{~nm}$ of $\mathrm{Ti}$ and $0.5 \mu \mathrm{m}$ of Au. The metal lines, each $50 \mu \mathrm{m}$ by $6 \mathrm{~mm}$ and spaced $250 \mu \mathrm{m}$ apart, were formed after lift-off.

To ensure removal of organics, the substrates were exposed to UV-ozone for $90 \mathrm{~min}$ immediately prior to wafer alignment. The alignment was such that the metal lines were orthogonally bonded to one another, forming $50 \times 50 \mu \mathrm{m}$ pads, as shown in Fig. 2. The bonding layer thickness thus formed was $1 \mu \mathrm{m}$. A vertical separation was maintained between the wafers by three triangular separators, each about $100 \mu \mathrm{m}$ thick and $1 \mathrm{~cm}$ long, until bonding. The wafers were bonded using Electronic Visions AB1-PV or EV-501 Bonder. The chamber was first purged with nitrogen and pumped to a slight vacuum. The temperature was then ramped up to the bonding temperature of either 260 or $300{ }^{\circ} \mathrm{C}$. Following a 3-min stabilization period, a small pressure was applied to allow the separators to be withdrawn and the bonding pressures were applied for the duration of the specified bond time, which varied from 2 to $90 \mathrm{~min}$. The bond pressure applied to the gold (as opposed to the wafer) were 30, 72, and $120 \mathrm{MPa}$. The temperature was subsequently ramped down.

The bonded wafers were diced into $8 \mathrm{~mm}$ wide strips, forming mechanical test specimens. A width-wise cut was made above the $\mathrm{KOH}$-etched notch to the DSP wafer, exposing the bonded interface. Mechanical tests were performed using a servo-hydraulic mechanical testing machine in its displacement-controlled mode at a rate of $0.075 \mathrm{~mm} / \mathrm{min}$. The specimen rested on the outer rollers, which sat on a fixture that was rigidly attached to the upward moving crosshead. The inner rollers were attached to the stationary portion of the testing machine 


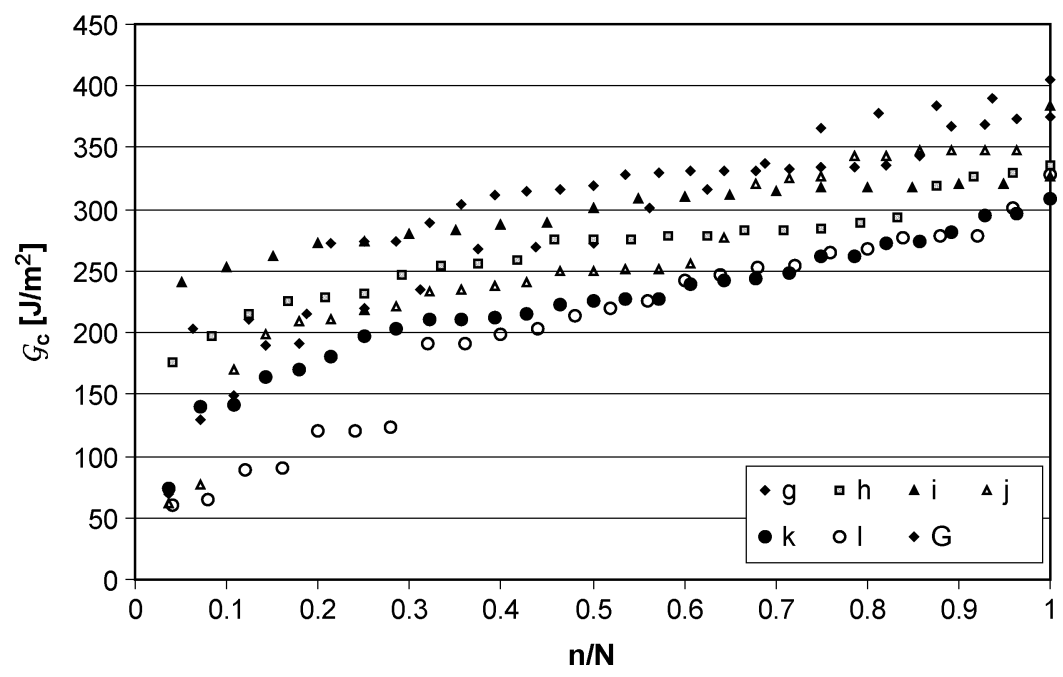

Fig. 3. $\mathcal{G}_{c}$ as a function of $\mathrm{n} / \mathrm{N}$ for 7 pairs of wafer bonded at $300{ }^{\circ} \mathrm{C}$ and $73 \mathrm{MPa}$ for $10 \mathrm{~min}$.

via an adjustable grip. The adjustable grip improved the positional leveling of the top apparatus. The alignment of the apparatus was checked immediately prior to testing with strain gauges located below the inner rollers. A $100 \mathrm{~N}$ load cell was used to monitor the load. Load and cross-head displacement data were captured by a LabVIEW program while real-time observations of the specimen were made with a long working distance microscope.

Once the test was completed, a width-wise line was scribed on the surface of the DSP wafer, on either side of the $\mathrm{KOH}$-etched notch and above the end of each of the cracks. The specimen was then loaded in a three-point bend jig to promote crack growth from the scribed line to the bond interface, thereby detaching the delaminated segments from the still bonded portion of the specimen. Since minimal force was required for this procedure, any observable deformation reflected those sustained during the four-point bend delamination test. The fracture surfaces were examined using a scanning electron microscope (SEM), optical microscope, and atomic force microscope (AFM).

\section{EXPERIMENTAL RESULTS AND DISCUSSION}

Equation (1) assumes that the bond extends the full width of the specimen. Since the bonding area in the specimens was discontinuous, the equation for calculating $\mathcal{G}_{c}$ must include the reduction in the bonding area

$$
\mathcal{G}_{c}=\frac{9\left(1-\nu^{2}\right)}{E}\left(\frac{P_{c} l}{b}\right)^{2}\left(\frac{1}{h_{1}^{3}}-\frac{1}{\left(h_{1}+h_{2}\right)^{3}}\right)\left(\frac{w_{S i}}{w_{A u}}\right)
$$

where $w_{S i}$ and $w_{A u}$ are the widths of the Si beam and the Au lines, respectively. The factor of 1.5 in (1) has become 9 in (2) to reflect the fact that the $50 \mu \mathrm{m}$ wide pads were separated $250 \mu \mathrm{m}$ apart along the length of the specimen as well.

The layout consisted of seven groups of lines, separated by approximately $2 \mathrm{~mm}$. Grids of bond pads thus formed when the wafers were aligned with lines orthogonally to one another, as schematically shown in Fig. 2. This pattern resulted in four separate sections within the inner rollers, two on either side of the notch. Due to the relatively large spacing between sections and changes in crack opening when the crack propagates through the spacing, the toughness of each of the sections can thus be calculated. The data presented henceforth contained four data points per specimen.

To obtain the cumulative distribution of $\mathcal{G}_{c}$, all the $\mathrm{N}$ data points were ranked in ascending order, with $n=\{1, \ldots, N\}$, and plotted with the corresponding value of $\mathcal{G}_{c}$. In addition, it is possible to match the failure morphology of a particular region with the measured toughness value. A cohesive failure occurs within the gold, whereas adhesive failure occurs at one of the $\mathrm{Ti}-\mathrm{SiO}_{2}$ interfaces, as determined by Auger spectroscopy.

\section{A. Repeatability}

The repeatability of the process and toughness measurement was studied with specimens bonded at $300{ }^{\circ} \mathrm{C}$ with an average applied pressure of $73 \mathrm{MPa}$ on the gold for $10 \mathrm{~min}$. Two pairs of wafers ( $\mathrm{g}$ and $\mathrm{h}$ ) were bonded using EV AB1-PV while the other five pairs used EV501 bonders. Specimens were tested in random order.

Failure was observed to occur predominantly within the gold layer. The majority of the $\mathcal{G}_{c}$ values fall between 200 and $350 \mathrm{~J} / \mathrm{m}^{2}$, as shown in Fig. 3. For specimens with $\mathcal{G}_{c}$ less than $100 \mathrm{~J} / \mathrm{m}^{2}$, large areas of poor, or possibly nonbonding, pads were found. As $\mathcal{G}_{\mathrm{c}}$ increases, so do the number of bonded pads and the degree of plastic deformation. At $\mathcal{G}_{c} \sim 300 \mathrm{~J} / \mathrm{m}^{2}$, nearly all of the pads were found to be bonded and plastic deformation was easily identified under an optical microscope. While there is no strong indication of tool dependence in the data, there is, however, significant scatter in $\mathcal{G}_{c}$. The scatter was not strongly correlated to variations in the process, such as the bonder chamber pressure, and the temperature at which the bond stack was placed and removed. Despite the large scatter in the $\mathcal{G}_{\mathrm{c}}$ per wafer, a comparable amount of scatter was found for any given wafer. Therefore, data from one or two wafers adequately capture the toughness trend for a given processing condition.

\section{B. Temperature and Pressure}

The effect of bonding temperature and pressure on the bond toughness were examined. In addition to the seven pairs of 

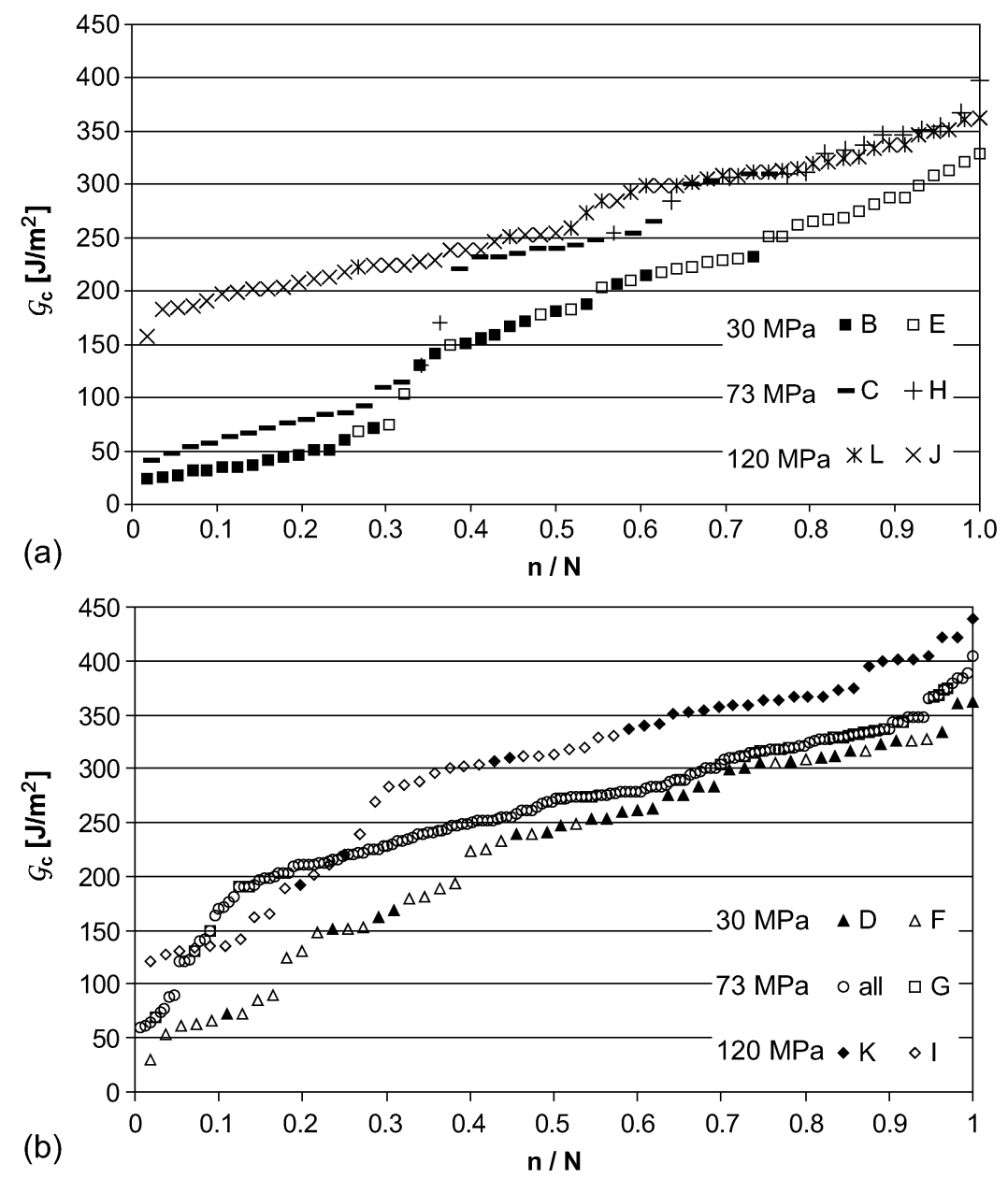

Fig. 4. $\mathcal{G}_{c}$ as a function of $\mathrm{n} / \mathrm{N}$. (a) Specimens bonded at $260{ }^{\circ} \mathrm{C}$. (b) Specimens bonded at $300{ }^{\circ} \mathrm{C}$.

TABLE I

BOND TEMPERATURE AND APPLIED PRESSURE (ON GOLD)

\begin{tabular}{c|c|c|c}
\hline Temp $\left[{ }^{\circ} \mathrm{C}\right]$ & $\mathrm{P}_{A u}[\mathrm{MPa}]$ & Wafer ID & e-beam run \\
\hline \multirow{3}{*}{260} & 30 & $\mathrm{~B} \& \mathrm{E}$ & $1 \& 2$ \\
\cline { 2 - 4 } & 73 & $\mathrm{C} \& \mathrm{H}$ & $1 \& 3$ \\
\cline { 2 - 4 } & 120 & $\mathrm{~J} \& \mathrm{~L}$ & $1 \& 3$ \\
\hline \multirow{3}{*}{300} & 30 & $\mathrm{D} \& \mathrm{~F}$ & $2 \& 3$ \\
\cline { 2 - 4 } & 120 & $\mathrm{I} \& \mathrm{~K}$ & $3 \& 2$ \\
\hline
\end{tabular}

wafers discussed in the previous section, two pairs of wafers were bonded using parameters shown in Table I.

Besides bond toughness, the percentage of bonded pads was also noted by examining the fractured surfaces. The fractured surface of pads that were bonded were rougher than the gold on either side of it, which was not bonded. The roughness is a result of plastic deformation, which was visible under an optical microscope. Highly deformed pads appeared dark. Hence, this visual contrast was used to assess whether a pad has been bonded or not. Digital images of the fractured surfaces of the two sections immediately on either sides of the notch were taken. A MATLAB program was then used to process the images and to count the percentage of pads that were successfully bonded. A pad was considered bonded if less than or equal to $10 \%$ of the (b)

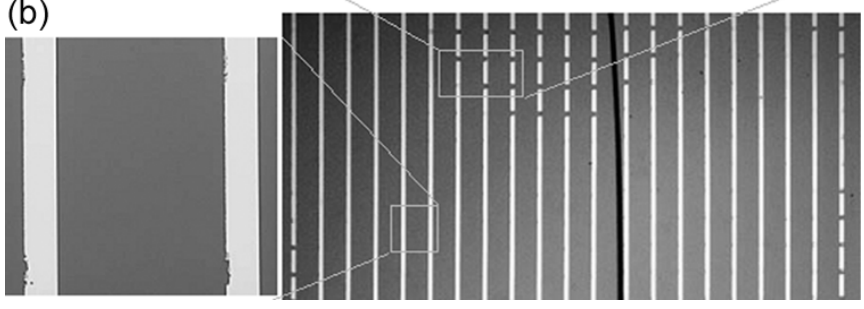

Fig. 5. Fractured surface of poorly bonded pads. (a) Region with adhesive failure. (b) Sign of deformation visible only at edge of the lines.

pixels in the pad were of the same greyscale or lighter ${ }^{1}$ than the surrounding gold.

Similar to results in Section IV-A, Fig. 4 shows that the toughness spans a wide range. In the low toughness region $\left(<100 \mathrm{~J} / \mathrm{m}^{2}\right)$, few cohesive failures and some adhesive

${ }^{1}$ Undeformed/unbonded Au is more reflective and appears brighter under optical microscope. 

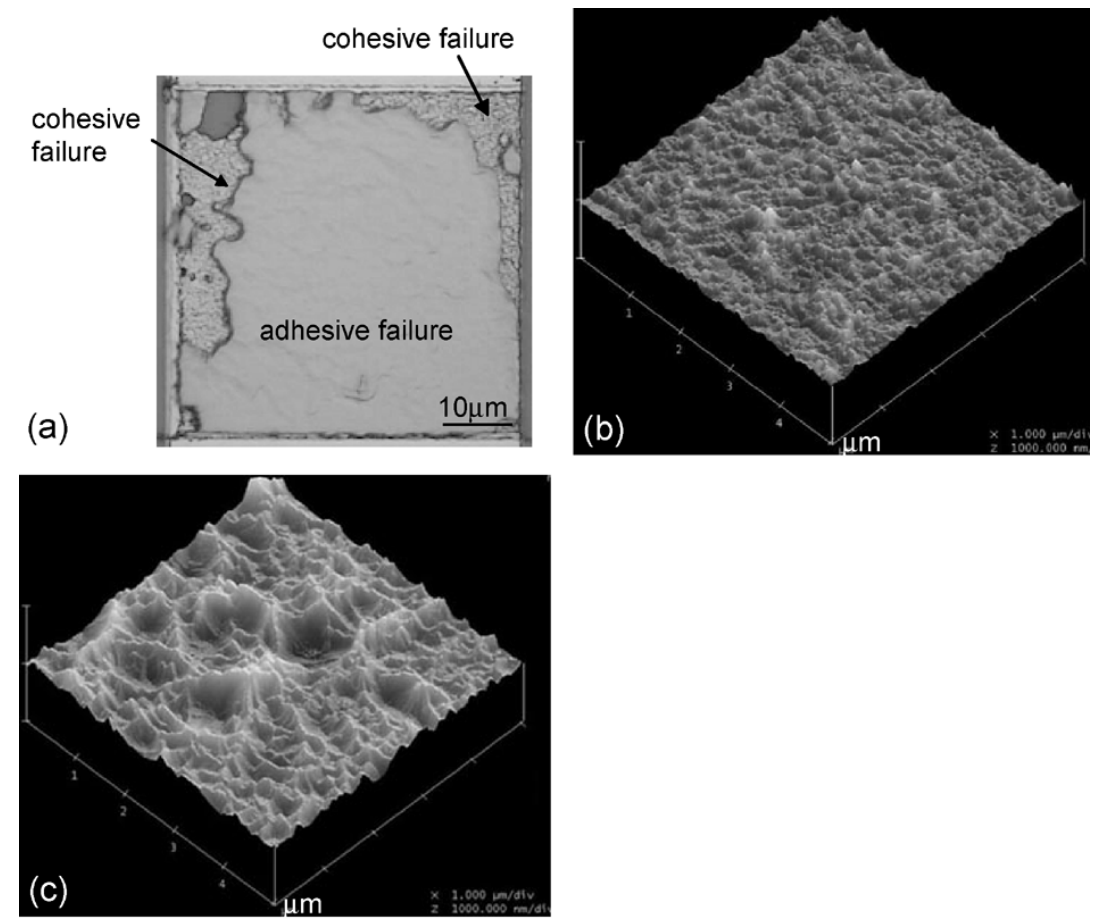

Fig. 6. Fractured surface of pads. (a) Optical micrograph of a pad with mostly adhesive failure and regions of visible plastic deformation. AFM images of a $5 \times 5 \mu \mathrm{m}$ area of specimens with (b) average $\mathcal{G}_{c}$ of $280 \mathrm{~J} / \mathrm{m}^{2}$ and rms of $36 \mathrm{~nm}$. (c) Average $\mathcal{G}_{c}$ of $352 \mathrm{~J} / \mathrm{m}^{2}$ and rms of $82 \mathrm{~nm}$.

failures were observed. The majority of the pads were poorly bonded, especially those bonded at the lowest values of temperature and pressure $\left(260{ }^{\circ} \mathrm{C}\right.$ and $\left.30 \mathrm{MPa}\right)$, and often the only indication of bonding was near the edges of the pads, as shown in Fig. 5. Little energy is thus required to delaminate the specimens. As the toughness increased, an increasing number of pads were found to exhibit either of the failure modes, indicating an improvement in the bond quality. Since more energy is dissipated in ductile deformation, higher toughness values were obtained from specimens with more cohesively failed pads. At the high end of the toughness range, above $200 \mathrm{~J} / \mathrm{m}^{2}$, most failures were cohesive with plastic deformation that was clearly visible under an optical microscope.

Toughness is expected to increase when the bonding temperature and pressure are raised. However, Fig. 4 shows that even those wafers bonded at $120 \mathrm{MPa}$ had some regions exhibiting low toughness. Similar to B and E specimens, a couple of specimens had large areas of poor or no bonding in the low toughness range $\mathcal{G}_{c} \leq 60 \mathrm{~J} / \mathrm{m}^{2}$. In the mid-to-lower toughness range, $60<\mathcal{G}_{\mathrm{c}} \leq 250 \mathrm{~J} / \mathrm{m}^{2}$, most of the pads were bonded. The relatively low toughness stemmed from the adhesive failure mode: less energy is dissipated in adhesive failure due to limited plasticity. In principle, adhesive failure should produce a fairly constant toughness, given it is an elastic energy release. But, experimental data of $\mathrm{Cu}$ bonded thin film stacks showed that the ductility of neighboring films allows additional energy to be dissipated, even when the failure mode was adhesive [10]. Hence, there is variation in the toughness measured, depending on the ratio of adhesively to cohesively failed bonds in a given specimen and the amount of energy dissipated in the process. Adhesive failures with varying degrees of plasticity were observed. Some are obvious, while others may be inferred, as shown in

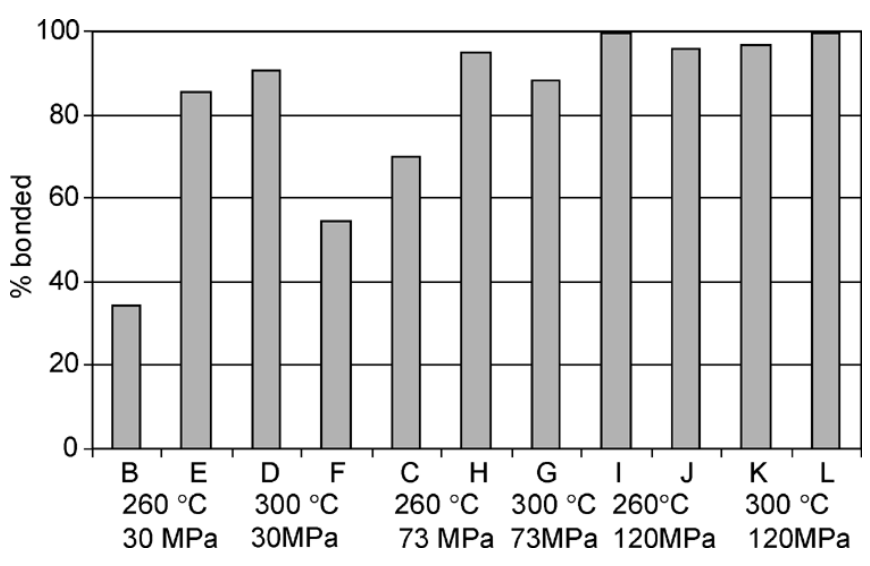

Fig. 7. Percentage of bonds that meet the bonding criterion.

Fig. 6(a). As the failure mode shifted toward being predominantly cohesive, the toughness increased. The plastic deformation also became more extensive, as shown in Fig. 6(b) and (c). For reference, the as-deposited gold has a rms roughness of $3 \mathrm{~nm}$.

Greater deformation may be obtained by either an increase in the applied pressure or creep deformation of the gold (due to an increase in temperature). The data support the expectation that increased toughness results when the bonding temperature and pressure are raised. Improved bond quality was evident in the toughness values and the observation of the extensive plastic deformation. The increase in the applied pressure, however, does not change the lateral dimensions of the pads, as illustrated in Fig. 6(a). The slightly enlarged areas of pads that failed adhesively in Fig. 5(a) is due to tearing of the pads, rather than increases in the footprint from the applied pressure. Therefore, the 


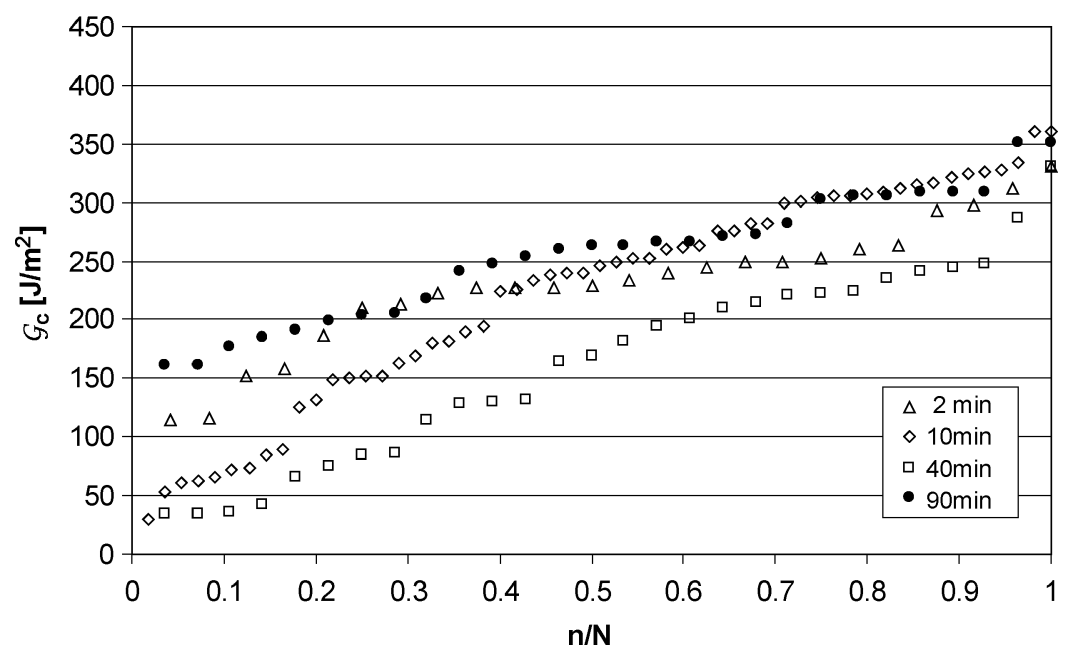

Fig. 8. $\mathcal{G}_{c}$ as a function of $\mathrm{n} / \mathrm{N}$ for specimens bonded for varying times.

dimensions of the bonding area is expected to be maintained up to $120 \mathrm{MPa}$. Results for the percentage of pads that were bonded, shown in Fig. 7, complement the toughness result. Data from most of the specimens from several bonding conditions merge and peak around $350 \mathrm{~J} / \mathrm{m}^{2}$. This suggests $300-375 \mathrm{~J} / \mathrm{m}^{2}$ as the upper bound of toughness that may be expected when bonding under the conditions studied.

\section{Time}

To explore the effect of bond time, wafers were bonded at $300{ }^{\circ} \mathrm{C}$ with an average applied pressure of $30 \mathrm{MPa}$ on the gold. The bond times were 2, 10, 40, and 90 min. Fig. 8 shows comparable $\mathcal{G}_{c}$ values regardless of the bonding time and the failure mode was predominantly cohesive failure. In contrast to the report of Drost et al. [6], where bond homogeneity improved when the bond time was greater than $1 \mathrm{~min},{ }^{2}$ the bond time was not found to affect significantly the toughness or the bond quality in the present paper. These are not contradictory results. The applied pressure is used to help bring surfaces together. Given the high mobility of gold and the elevated temperature at which the substrates were held, bonds can form relatively quickly as a result of surface diffusion. Therefore, improvement in the bond quality was not observed in the range examined in the current study.

\section{Finite ELEMENT ANALYSIS}

Process variations alone cannot explain the amount of scatter in the $\mathcal{G}_{c}$ values. Moreover, nonuniform deformation was found on the fractured surfaces. Better bonding, in terms of increased deformation, was found more frequently on the pads that were located around the edges of the sections. This observation led to the hypothesis that pressure was not uniformly distributed across the wafer during bonding. The local pressure is an important parameter in thermocompression bonding. It aids in overcoming some of the barriers imposed by the physical system, such as wafer waviness, and thickness and roughness variations, so that the gold surfaces may be brought into close proximity for bonding to take place. A finite element model was built to better

\footnotetext{
${ }^{2}$ The bond time range was not mentioned.
}

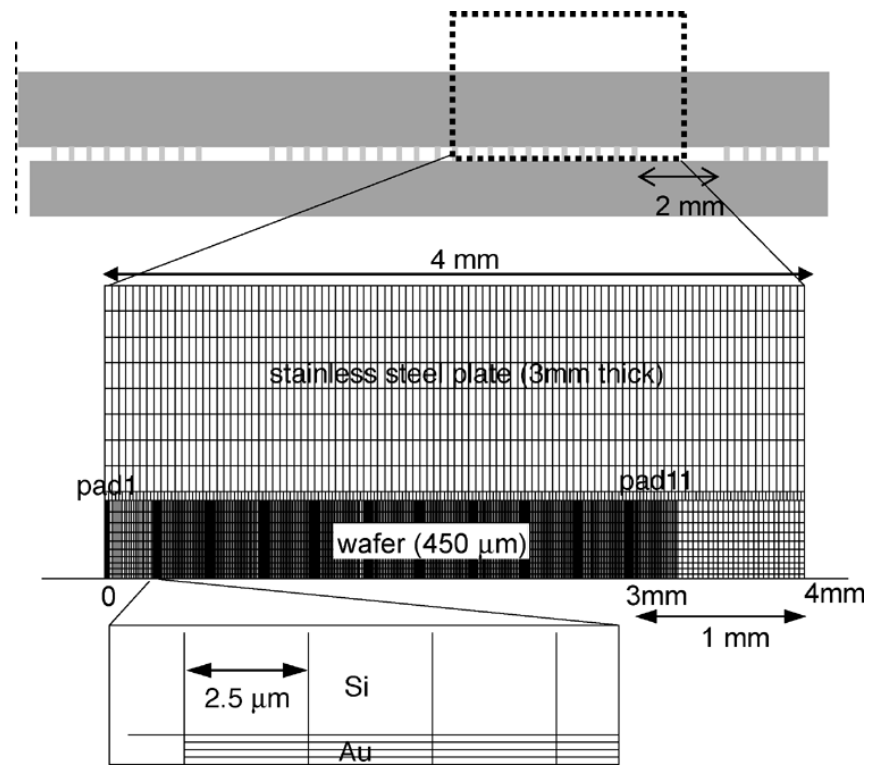

Fig. 9. Mesh of the area modeled.

understand how nonuniform pressure may arise, due to the effect of patterning.

\section{A. Model Description}

The bonding process was simulated using commercial finite element software, ABAQUS, with its standard solver package [12]. A 2-D model $4 \mathrm{~mm}$ wide, which encompassed half of a $21 \times 21$ pad section, was created with 8-noded plane strain elements. Symmetric boundary conditions in the x-direction were imposed on both ends of the structure. The mesh included the 3-mm-thick stainless steel diffusion plate that was placed on the wafer during the bonding operation, which is only partially shown in Fig. 9. The Young's modulus and Poisson's ratio of the stainless steel are $193 \mathrm{GPa}$ and 0.3 , respectively. The wafer is $450 \mu \mathrm{m}$ thick, with elastic properties of $169 \mathrm{GPa}$ and 0.22 for the Young's modulus and Poisson's ratio, respectively. The $50 \mu \mathrm{m}$ wide, $0.5 \mu \mathrm{m}$ thick gold pads were modeled as elastic-perfectly plastic: the Young's modulus, Poisson's ratio and the yield stress are $70 \mathrm{GPa}, 0.42$, and $120 \mathrm{MPa}$, respectively. The uniaxial yield 


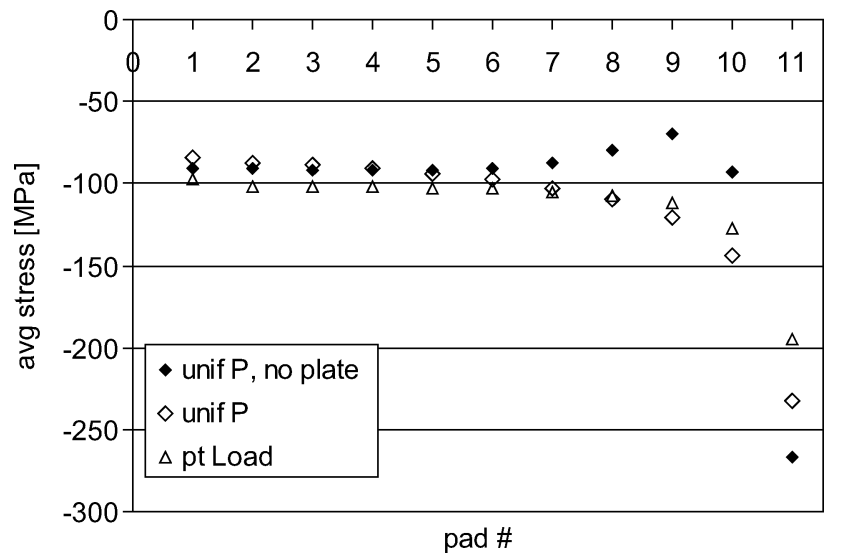

Fig. 10. Simulation result of average pressure per pad.

stress of $120 \mathrm{MPa}$ was chosen in order to simulate the maximum pressure applied in the experiments.

The bottom of the pads were fixed in the y-direction to simulate bonding with the gold on the other wafer, which was not modeled, but represented by a rigid line. Three boundary conditions were simulated: uniform pressure applied to the wafer (with a structure that contained only the wafer and gold pads), uniform pressure applied on the stainless steel plate, and a point load applied at the topmost, left corner node of the stainless steel plate. This last condition simulates a worst case condition of nonuniform loading. In all cases, the resultant mean pressure on the gold pads is $120 \mathrm{MPa}$. Each gold pad is formed by 20 columns and 4 rows of elements. The stress on the pad was obtained at the centroids of the elements located on the bottom row of each column.

\section{B. Simulation Results}

In the model, the left edge of pad 1 is coincident with the axis of symmetry while pad 11 is closest to the diesaw alley. Fig. 10 shows that the average stress per pad is not uniform from the center of the section to the edge where the diesaw alley is located. The edge pads experienced much higher stress than the ones located near the center, which is in agreement with experimental observations. Moreover, on average, all three boundary conditions resulted in pads experiencing lower pressure than the nominal applied pressure of $120 \mathrm{MPa}$. The higher pressure exerted on the edge pads was due to the $2 \mathrm{~mm}$ gap that separates sections of bond pads. Fig. 11 shows the displacement of the wafer at the end of the structure $(\mathrm{x}=0.3$ to $0.4 \mathrm{~mm})$ that was not supported by gold pads underneath. The parabolic profile indicates bending in the wafer. Depending on the boundary condition, the wafers could touch near the center of the $2 \mathrm{~mm}$ gap. The coincidence of the point load case with the uniform pressure cases indicates that the stainless steel plate should be sufficient to spread the applied load.

Experimentally, evidence of wafers touching near the center of the diesaw alley was observed. This matches the result of the simulation of uniform pressure applied directly on the wafer, without the stainless plate. In reality, though, there was a stainless steel plate that pressed against the wafer stack. The more representative boundary conditions were ones with the stainless

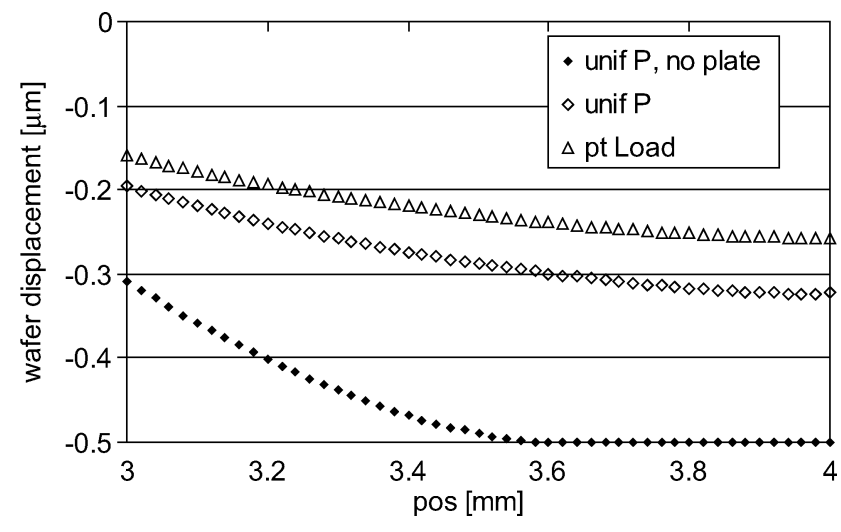

Fig. 11. Displacement of the wafer in the diesaw alley.

steel present. While the simulation results also showed bending of the wafer, the displacement was insufficient for the wafer to touch. However, the model assumed a perfectly uniform gold thickness across a perfectly flat wafer that was pressed by a perfectly flat stainless steel plate. In actuality, the gold thickness is not uniform, wafer thickness variations and waviness exists, and the applied pressure is not perfectly uniform across the wafer by the bonding tool itself. These factors can cause pressure to redistribute and would be likely to produce an additional displacement.

The validity of using this simple 2-D model to simulate a 3-D phenomenon was considered by Moon [13]. A 3-D model representing a quarter of the whole wafer was built. In Moon's model, the wafer and gold pads were modeled as shell and spring elements, respectively. To reduce the number of elements, the stainless steel plate was not modeled. The global response obtained from a uniform pressure condition was then fed into a local model, which consisted of approximately $1 / 4$ of the section $(10 \times 10$ pads $)$. The 3 -D result matched very well with the result obtained from the simple 2-D model without the stainless steel plate. Fig. 12 also shows a nonuniform pressure distribution across the pads, with the edge pads experiencing higher pressure than the ones in the center. Because of the boundary condition, the lowest pressure was found in the pads immediately neighboring the edge pads, as in the case of the 2-D simulation. The results support, qualitatively, the observation that pads tend to be better bonded near the edges of the sections.

More complicated models must be constructed in order to fully understand the source of the nonuniform bonding observed experimentally. The results of the simple 2-D model presented in this section indicates that some of the nonuniformity may be a result of the layout, where long sections of the wafer were not supported by the gold. Therefore, the wafers undergoes bending during bonding under high applied pressure. This in turn results in a nonuniform pressure distribution across the wafer.

\section{GUIDELINES FOR THERMOCOMPRESSION BONDING}

Experimental results demonstrated that bonding can be achieved at $260{ }^{\circ} \mathrm{C}$, well within the acceptable range for packaging applications. Although bonds can form at relatively low pressures [7], the uniformity and yield are critical considerations for implementation in wafer-level packaging. Based 


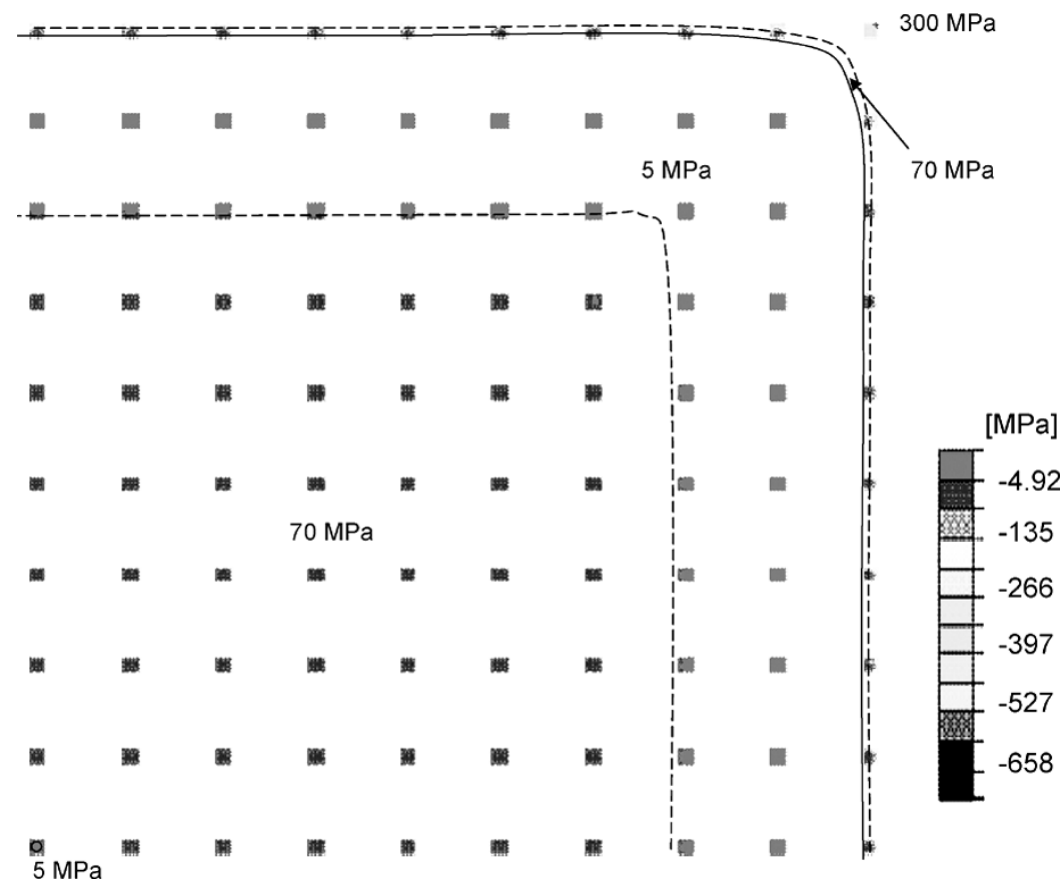

Fig. 12. Simulation result of the 3D model of the stress distribution on each pad.

on the data presented in this paper, the following processing recommendations are made:

- The gold film should be deposited on diffusion barrier films such as oxide [7]. Nitride should be equally effective. In previous work, silicon diffusion to the gold surface was found to be detrimental to bonding.

- As a process that forms bonds when clean metal asperities touch, the e-beam deposition process needs to be controlled to prevent source spitting. While the test structure used in the studies resulted in a relatively flat surface, greater flatness deviations will be present in actual devices. It is believed that the process can tolerate surface topography, provided the bonding material is deposited at the highest locations and that wafer curvature is minimized whenever possible.

- Surface treatment to remove organics, such as the UV-ozone exposure used in this paper, is recommended.

- Typically, low processing temperature and pressure are preferred. Based on the results presented in Fig. 7, bonding temperature and pressure of $260{ }^{\circ} \mathrm{C}$ and $120 \mathrm{MPa}$ are recommended to ensure the level of repeatability and yield desired in manufacturing environments. Since the pressure may cause damage to the underlying thin films or other structural materials, a tradeoff between pressure and temperature can be made. The decision depends on individual device constraints and optimal parameters have to be developed.

- The bond time was not shown to affect significantly the bond quality. Given the preference for faster throughput, the bond time can be as short as $2 \mathrm{~min}$.

- While wide seal rings waste valuable space on the wafer, rings that are too narrow may reduce reliability of the package. Linewidth on the order of $50 \mu \mathrm{m}$ is a good starting point for initial process development.
The linewidth was observed to be preserved under the conditions presented in this paper.

- Finally, the layout should be designed to have more evenly spaced features to avoid geometry-induced pressure nonuniformity. Dummy support structures may be incorporated.

\section{CONCLUSION}

It was expected that higher deformation of the gold would lead to improved bonding. Indeed, the overall trend of the data showed increased toughness with increasing bonding temperature or pressure. In addition, the plastic energy dissipation increased with the bonding parameters. For specimens bonded at $7 \mathrm{MPa}$ or less, the plastic deformation was visible only under SEM [7]. In contrast, much of the deformation in the specimens reported in this paper was visible using an optical microscope under low magnification.

The exact correlation of temperature or pressure to $\mathcal{G}_{c}$ was difficult to define given the large scatter observed. The scatter does not appear to be related to process variations such as initial temperature of the bonder, background pressure during the gold deposition, delays between UV-ozone clean and alignment. However, the range of scatter shown per wafer is fairly representative of the scatter that could be expected for the given bonding condition. Ductile fracture can involve much more energy than that required to break the bonds at the interface due to the ability to dissipate energy by plastic deformation. There are several mechanisms by which plastic energy is dissipated: directly as in visible ductile fracture within the bulk gold, indirectly in the gold even though the eventual fracture occurs adhesively, or in combination. In many of the systems studied by other investigators, interfacial (adhesive) failures were found [10], [11]. Both adhesive and cohesive failures were observed in the specimens tested in the present paper. The prediction of 
the crack path is complicated and was not pursued in this paper. Only experimental observations were noted. It was found that when a lower value of $\mathcal{G}_{c}$ was measured, the specimens tended to fail adhesively while higher values of $\mathcal{G}_{c}$ corresponded to cohesive failures.

The pressure distribution during the bonding process was modeled using finite element analysis. The simulation results showed that the mask layout produced an uneven distribution of pressure-higher on pads located near the edges than ones near the center. This nonuniformity was most noticeable experimentally in bonds produced under lower bonding pressures. Moreover, this was one of the main contributors to the bond toughness variation found. Finally, some guidelines for wafer-level process bonding were presented.

\section{ACKNOWLEDGMENT}

Fabrication was carried out at MIT Microsystems Technology Laboratories and tested at MIT Technology Laboratory for Advanced Composites. The 3-D model analyzed by H.-S. Moon is gratefully acknowledged.

\section{REFERENCES}

[1] R. F. Tylecote, The Solid Phase Welding of Metals. New York: St. Martin's, 1968

[2] L. W. Condra, J. J. Svitak, and A. W. Pense, "The high temperature deformation properties of gold and thermocompression bonding," IEEE Trans. Parts Hybrids Packag., vol. PHP-11, pp. 290-296, 1975.

[3] Y. G. Kim, J. K. Pavuluri, J. R. White, I. J. Busch-Vishniac, and G. Y. Masada, "Thermocompression bonding effects on bump-pad adhesion," IEEE Trans. Comp. Packag. Manufact. Technol., vol. 18, pp. 192-199, 1995.

[4] L. Parameswaran, A. Mirza, W. K. Chan, and M. A. Schmidt, "Silicon pressure sensors using a wafer-bonded sealed cavity process," in Proc. 8th Int. Conf. Solid-State Sensors Actuators, vol. 2, 1995, pp. 582-585.

[5] B. K. Furman and S. G. Mita, "Gold-Gold (Au-Au) thermocompression (TC) bonding of very large arrays," in Proc. 42nd Electronic Components and Technology Conf., 1992, pp. 883-889.

[6] A. Drost, G. Klink, S. Scherbaum, and M. Feil, "Simultaneous fabrication of dielectric and electrical joints by wafer bonding," in Proc. SPIE-Micromachined Devices and Components IV, 1998, pp. 62-71.

[7] C. H. Tsau, S. M. Spearing, and M. A. Schmidt, "Fabrication of waferlevel thermocompression bonds," J. Microelectromech. Syst., vol. 11, pp. 641-647, 2002.

[8] W. P. Maszara, G. Goetz, A. Caviglia, and J. B. McKitterick, "Bonding of silicon wafers for silicon-on-insulator," J. Appl. Phys., vol. 64, pp. 4943-4950, 1988.

[9] P. G. Charalambides, J. Lund, A. G. Evans, and R. M. McMeeking, "A test specimen for determining the fracture resistance of bimaterial interfaces," J. Appl. Mech., vol. 56, pp. 77-82, 1989.

[10] M. Lane, R. H. Dauskardt, A. Vainchtein, and H. Gao, "Plasticity contributions to interface adhesion in thin-film interconnect structures," $J$. Mater. Res., vol. 15, pp. 2758-2769, 2000.

[11] Q. Ma, "A four-point bending technique for studying subcritical crack growth in thin films and at interfaces," J. Mater. Res., vol. 12, pp. 840-845, 1997.

[12] Hibbitt, Karlsson, and Sorensen, Inc., ABAQUS Version 6.3.

[13] C. H. Tsau, Fabrication and characterization of wafer-level gold thermocompression bonding, MIT, Cambridge, 2003.

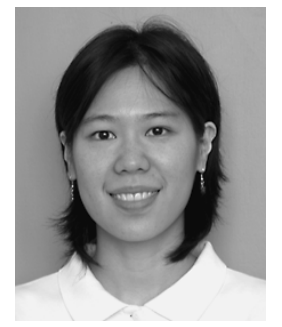

Christine H. Tsau received the S.B. and Ph.D. degrees in materials science and engineering from the Massachusetts Institute of Technology (MIT), Cambridge, in 1998 and 2003, respectively.

She was a Semiconductor Research Corporation Graduate Fellow. Currently, she is a Postdoctoral Associate with the Biological Engineering Division of MIT. Her current research focuses on bioMEMS devices.

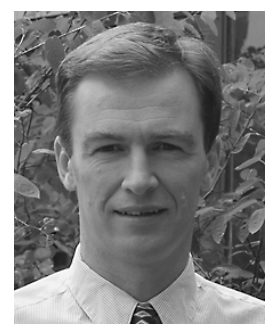

S. Mark Spearing received the Ph.D. degree from the Engineering Department, Cambridge University, in 1990 .

$\mathrm{He}$ is a Professor of aeronautics and astronautics at the Massachusetts Institute of Technology (MIT), Cambridge, where he has been since 1994. Since 2004, he has been a Professor of engineering materials with the University of Southampton, U.K. His technical interests include materials characterization and structural analysis and design of MEMS, development of wafer bonding-technologies, microelectronic and MEMS packaging, and advanced composites. Since 1995, he has been responsible for materials, structural design, and packaging tasks of the MIT MicroEngine, MicroRocket, Micro-Chemical Power, and MicroHydraulic Transducer projects, as well as conducting cross-cutting underpinning technology development.

Dr. Spearing is an Associate Editor of the JOURNAL OF MicROELECTROMECHANICAL SYSTEMS and a Member of the American Society of Mechanical Engineers (ASME).

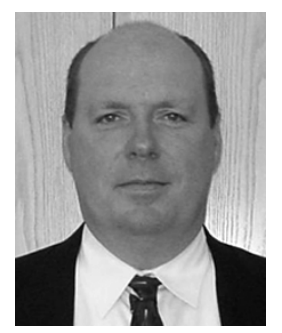

Martin A. Schmidt (S'88-M'85-SM'00-F'03) received the B.S. degree in electrical and computer engineering from Rensselaer Polytechnic Institute, Troy, NY, in 1981 and the S.M. and Ph.D. degrees in electrical engineering and computer science from the Massachusetts Institute of Technology (MIT), Cambridge, in 1983 and 1988, respectively.

He investigates microfabrication technologies for integrated circuits, sensors, and actuators; design of micromechanical sensor and actuator systems; mechanical properties of microelectronic materials, with emphasis on silicon wafer bonding technology; integrated microsensors; and microfluidic devices. His current research projects involve novel applications of MEMS technologies to a variety of fields, including miniature gas turbines, miniature chemical reactors, microswitches, biological applications, and sensors monolithically integrated with electronics. His work has been sponsored by NSF, ARO, SRC, AFOSR, NIH, and a host of industries. He is a participant in the educational and research activities of the Cambridge-MIT Institute (CMI). He has more than 150 refereed technical publications and 29 patents/patent applications.

Dr. Schmidt is recipient of a number of awards including the Ruth and Joel Spira Teaching Award, the NSF Presidential Young Investigator Award, and an honorary doctorate from the Technical University of Denmark. 